\title{
EAl Endorsed Transactions

\section{Investigating the Digital Literacy Needs of Healthcare Students when using Mobile Tablet Devices}

\author{
George Evangelinos ${ }^{1, *}$ and Debbie Holley ${ }^{2}$ \\ ${ }^{1}$ Anglia Ruskin University, Faculty of Health Social Care and Education, East Road, Cambridge, United Kingdom, CB1 1PT \\ ${ }^{2}$ Bournemouth University, Executive Business Centre, 89, Holdenhurst Road, Bournemouth, BH8 8EB
}

\section{Abstract}

This paper presents the findings of two case studies that investigated the digital attitudes, skills and development needs of healthcare students when using mobile tablet devices to assess student-nurse competencies in clinical practice and when used in the classroom to facilitate digitally enabled learning. Participants have been asked to complete a bespoke scenariobased digital competence self-assessment questionnaire based on the EU DIGCOMP framework; this enabled a baseline for individual and group practice. The first case study documented the individual perceptions and experiences on the digital literacy of students by analysing the students' reflective diaries. In the second a questionnaire documented the student experience of utilising tablet devices to support the delivery of technology-enhanced learning designs in the classroom. The results showed a complex, highly-individual profile for each student, while the group exhibited some common trends and characteristics. The majority of students felt capable in using tablet technologies for learning but there was no clear consensus as to whether technology-enhanced learning should be introduced on a larger scale. Further work is proposed to model a curriculum-development process for embedding digital literacies into curriculum delivery through the utilisation of technology-enhanced, activity-based learning designs.

Keywords: digital competence, digital literacy, EU DIGCOMP framework, mobile devices, learning design

Received on 31 March, 2016, accepted on 31 March, 2016, published on 11 April, 2016

Copyright (C) 2016 G. Evangelinos and D. Holley, licensed to EAI. This is an open access article distributed under the terms of the Creative Commons Attribution licence (http://creativecommons.org/licenses/by/3.0/), which permits unlimited use, distribution and reproduction in any medium so long as the original work is properly cited.

doi: 10.4108/eai.11-4-2016.151155

\section{Introduction}

Two case studies have been conducted to investigate the digital literacy needs of students and document their experiences of using tablet devices in healthcare education. The first case study was conducted within the boundaries of an assessment improvement project where tablet devices were used to record electronically a portfolio of the assessed practice competences of studentnurses. The student perceptions and experiences on digital literacy have been documented in reflective diaries. The second case study was conducted with a group of Midwifery students that had been asked to complete technology-enabled learning activities in the classroom by using mobile tablet devices. The activities were designed

"Corresponding author. Email: george.evangelinos@anglia.ac.uk to deliver curriculum learning outcomes and at the same time incorporate a variety of digital skills. A short student-experience questionnaire was used to evaluate the student experience of undertaking these technologyenhanced learning activities. In both case studies a bespoke digital literacy, self-evaluation questionnaire that was aligned to the EU DIGCOMP framework [4] was used to baseline the digital competences of the students.

The digital-literacy work is part of a wider action research project that has validated the suitability of the DIGCOMP digital literacy framework for use in healthcare education through a qualitative analysis of the views of students and staff [1], [2], developed selfassessment tools for quantitative assessing and mapping of their digital competences [3], and documented the views of students about the delivery of digital-literacy 
skills embedded within the curriculum delivery by utilisation of technology-enhanced activities designed along Dalziel's [4] Learning Design principles.

\section{Methodology}

In both case studies participants completed a bespoke skills-based online digital competence self-assessment questionnaire that allowed base-lining of the digitalliteracy competence level of the groups. This questionnaire was based on the EU DIGCOMP framework [5] and included 21 questions organised into 5 themes. This is the body text with indent.

\section{Table 1 - DIGCOMP Framework Competence Areas}

\section{DIGCOMP Framework Digital Competences}

1. Information

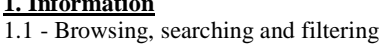

information

1.2 - Evaluating information

1.3 - Storing and retrieving information

3. Content creation

3.1 - Developing conten

3.2 - Integrating and re-elaborating

3.3 - Copyright and licences

3.4 - Programming

\section{Communication}

2.1 - Interacting through technologies

2.2 - Sharing information and content

2.3 - Engaging in online citizenship

2.4 - Collaborating through digital

channels

2.5 - Netiquette

2.6 - Managing digital identity

4. Safety

4.1 - Protecting devices

4.2 - Protecting personal data

4.3 - Protecting health

4.4 - Protecting the environment

\section{Problem solving}

5.1 - Solving technical problems

5.2 - Identifying needs and

technological responses

5.3 - Innovating and creatively using

technology

5.4 - Identification of digital

competence gaps

The questionnaire toolkit required the participants to self-assess their digital competences by selecting the most appropriate scenario to their perceived skill set. Evangelinos and Holley [2] found that the student population has diverse digital skills, and attitudes towards technology and prior experiences. Students were asked to think whether they possessed the skills and attitudes to complete the proposed activities regardless of having actually completed similar activities in the past. The questionnaire presented the participants with 5 competence areas expressed as groups of questions. Each question presented the participants with 4 examples of possible hypothetical role-play technology-use scenarios and asked them to select the answer that best matched their skills. The scenarios were progressively becoming more complex and were designed to represent different digital literacy profiles ranging from lack of skills to elementary, intermediate and advanced. The scenarios were customised to present the students with authentic situations relevant to their academic experiences. An example of the scenario-based questions can be seen in Figure 1 - Question 2.4 of the DIGCOMP Selfassessment Toolkit below.

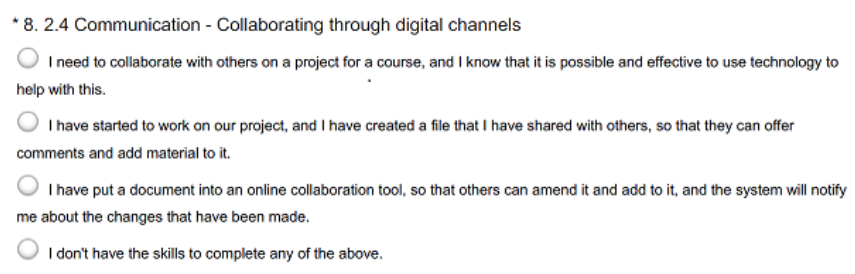

Figure 1 - Question 2.4 of the DIGCOMP Selfassessment Toolkit

The questionnaire also included a basic demographic section concerning the participants' gender and age and two additional questions on how they find out about new technologies and if they have used a number of preselected technologies for formal/informal learning, research, work and in their personal life.

In the first case study 24 out of 30 nursing students that participated in the sustainable electronic assessment project completed the questionnaire (return rate of $80 \%$ ). In the second case study 36 out of 101 midwifery students participating in the Classroom Learning Design interventions completed the questionnaire (return rate of $36 \%$ ). The results were exported and analysed by using the Microsoft Excel 2010 spreadsheet software. For the purposes of this paper the group characteristics of the students will be examined. A wealth of quantitative indicators of student digital-behaviour was revealed. The questions for each competence area were averaged together for each student group to give a more reliable single number index. The indices for each group are as per Error! Reference source not found. below.

The group digital-literacy map presents the averagegroup digital-literacy index for each group as a composite index deriving from the mean average across a number of competence-specific scenarios. Note that each area is quantified by different numbers of scenarios in (3-6-4-44). For example, the information-area index is a composite mean average of three information-literacy sub-questions; the communication area is expressed as the mean average of six sub-questions and content creation; safety and problem solving are represented by the mean average of four questions each.

For the Sustainable Electronic Assessment (SEA) case study students were invited to complete short reflective diaries to reflect and record their technology-use experiences in their private, academic and work lives. Students were asked to reflect and report in these diaries their perceptions of digital literacy, comment on the views concerning the acquisition of skills and areas in need of further development, and provide feedback suggestions on how the university can facilitate the enhancement of their digital skills. 15 students out of 30 completed the reflective diaries corresponding to a significant percentage $(50 \%)$ of the participants. The analysis was conducted by using QSR NVivo 10 software and coding the reflective diaries into themes following the Glaser and Strauss' [6] Grounded Theory approach, as well as the 
coding recommendations by Miles and Huberman [7] and Guest et al [8].

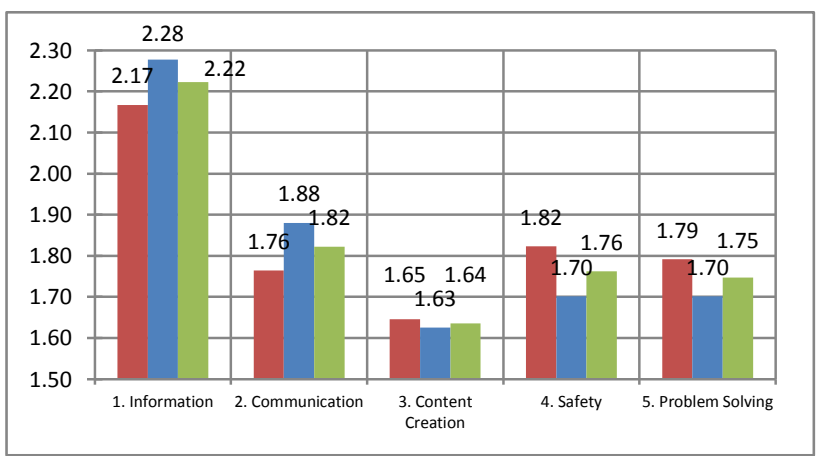

Figure 2 - Digital Literacy Group Indices

Explanations of pertinent ethical considerations, such as confidentiality of collected data, anonymity of the subjects, ownership of the data, and results of the study were provided and the participants were given the choice of participating anonymously, withdrawing without penalties or even dictating conditions on the use of data. Informed consent was obtained in writing according to the research protocol governed by the institution's ethical procedures.

In the second case study the student experience was investigated further by experimenting with technologyenhanced, activity-based learning designs delivered through a combination of pre-classroom work and inclassroom activities. The chosen module was taught by a number of tutors under the co-ordination of the module leader in two different campuses. In order to maintain parity of the student experience two student groups were formed based on the geographical location of their studies. Both groups were given exactly the same information and were subjected to the same research protocol. The research was conducted according to the institution's ethical procedures dictating the confidentiality of collected data, retaining the anonymity of the subjects and specifying the parameters around the ownership of the study and how the data could be used. Informed consent was provided electronically by the participants agreeing to participate after they had a chance to review the research protocol. Participants were also given the choice of participating anonymously, withdrawing without penalties or even dictating conditions on the use of data. It must be noted that participation in the learning activities was compulsory as it was an integral part of the module but it was made clear to the students that participating in the research and answering the two research questionnaires was optional.

The learning designs utilised in this experiment were co-designed with the module leader and comprised of 3 sessions that were delivered twice (once in each geographical location) to 101 students in total. The learning activities were re-designed to meet two requirements:

a) Changes in assessment and staffing that necessitated changes in the mode of curriculum delivery

b) The need to enhance the digital capabilities of students.

A brief description of the activities mapped against the high-level digital literacy areas can be seen in Table $2-$ Activities Mapped against the DIGCOMP ${ }_{\text {v. } 1}$ Framework Digital Competence Areas below.

\section{Table 2 - Activities Mapped against the DIGCOMP ${ }_{\mathrm{v} .1}$ Framework Digital Competence Areas}

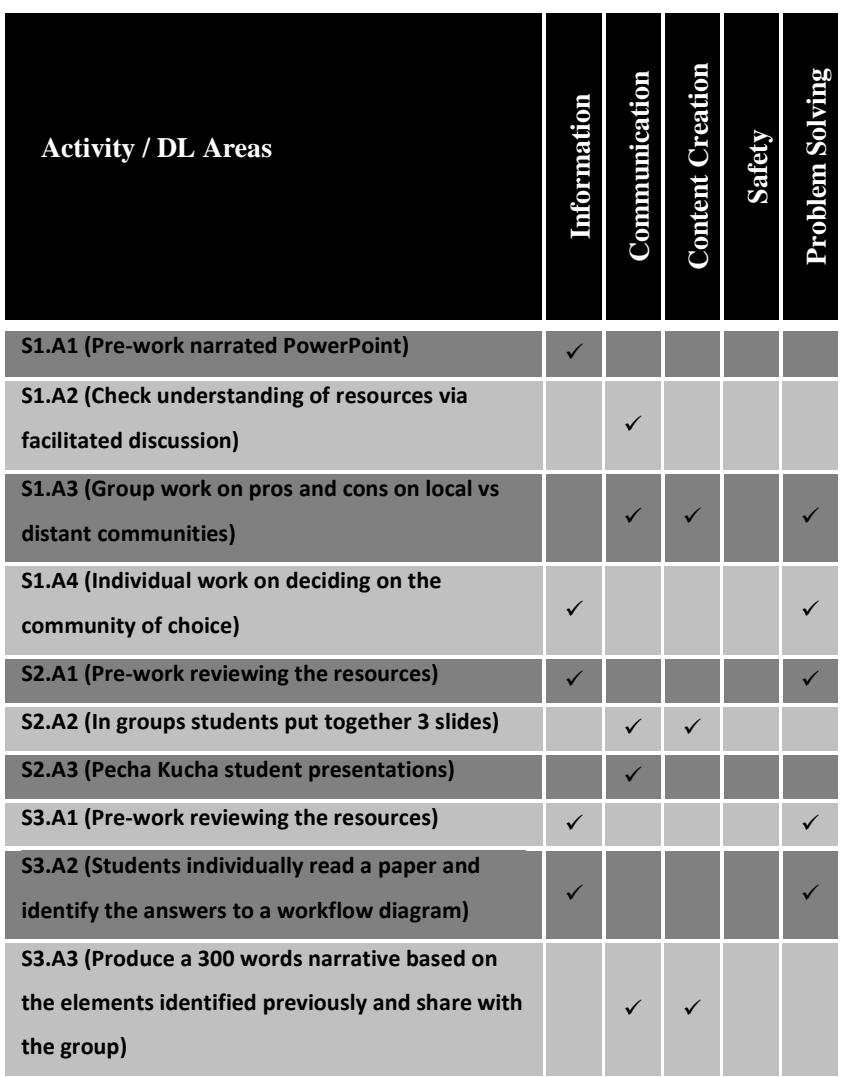

The digital component of the activities was delivered through a combination of institutional technologies and utilisation of cloud-based services, such as Google Documents and Google Drive. The selection of the technologies used was determined by the availability of the institutionally-supported technologies and the logistics of ensuring a sufficient number of digital devices were available to students who might not have owned their own. A pool of institutionally owned tablet devices was used to ensure that every student had access to a digital device to carry out the activities although students had been encouraged to use their own devices if they wished to do so. 
The student experience was evaluated by issuing a short questionnaire to document the student experience at the end of the third session and was completed by 56 out of the 101 students corresponding to a return rate of $55 \%$. Students were asked to indicate their agreement on a fourstep agreement/disagreement scale that included an option for not wanting to answer for the following statements:

- I enjoy working collaboratively

- I learn better by engaging in activity-based learning

- I feel that I have the digital skills required to complete the activities

- I need more support in using technologies

- I would like to have more opportunities to learn collaboratively and participate in activity-based, technology-enhanced learning

These statements were used to evaluate the student experience of undertaking the above mentioned technology-enhanced learning activities.

\section{Results}

The self-assessment questionnaire used to baseline digital literacy was completed by 57 respondents in total where $55(96 \%)$ were female and $2(4 \%)$ male. From these 34 $(60 \%)$ were between $18-25$ years of age, $14(24 \%)$ between 26-35 years of age, 5 (9\%) between 36-45 years of age and $4(7 \%)$ between $46-55$ years of age. The age range distribution of the participants across both casestudies can be seen in Figure 3 - Participant Age Range Distribution below.

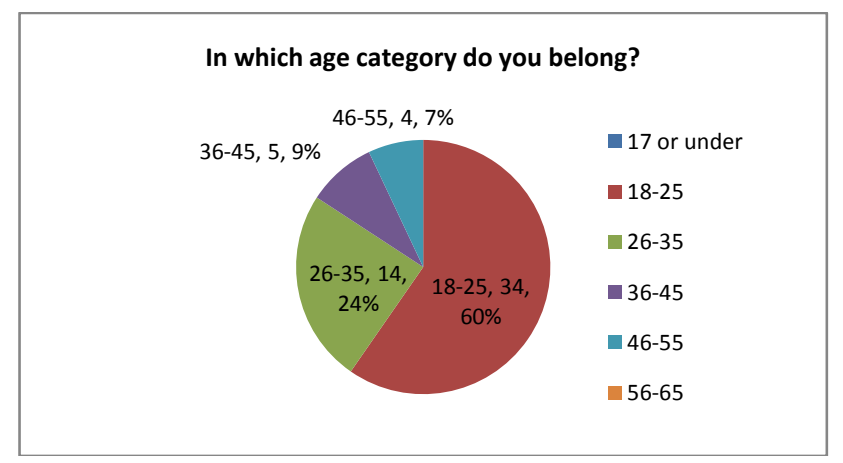

\section{Figure 3 - Participant Age Range Distribution}

The 21 questions (organised in the 5 competence areas) define 5 key metrics: a) Information b) Communication c) Content Creation d) Safety and Privacy and e) Problem Solving (see: Error! Reference source not found.). For example, the Information-literacy composite index with an average of (2.22) points (on a scale from 0-3 where 0 means no skills, 1 is basic, 2 intermediate and 3 is considered as advanced) denotes that on average students have just over an intermediate self-declared competency in the information competence area. The groups were least confident about their self-declared skills in the Content- Creation competence area with an average score of (1.64), or basic competence. The average value of each digital-literacy area can be used to baseline the general group competency in this area and, when combined, give a single value index of the group's digital-literacy potential. The frequency distribution gives a more nuanced perspective on the numbers of individuals at each level of competency across the digital-literacy competence areas.

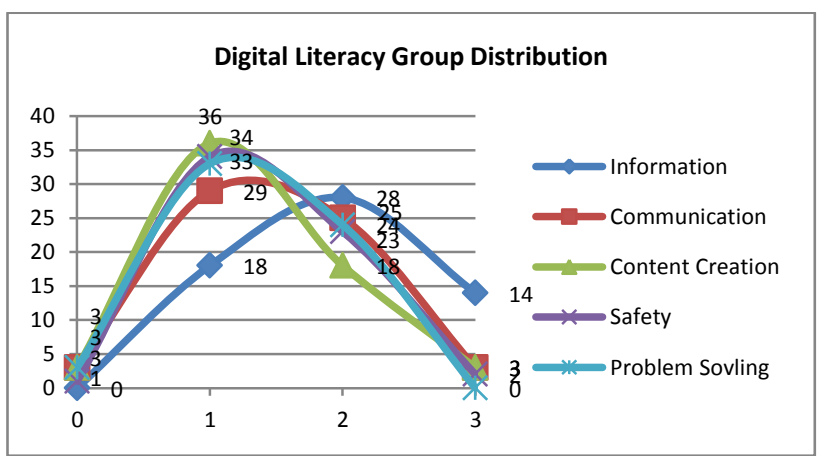

Figure 4 - Digital Literacy Group Distribution

As it can be observed in Figure 4 - Digital Literacy Group Distributio above the capability across the different areas of digital literacy of the individuals within the groups is variable. On average the group information and communication capabilities were more developed with the majority of students self-assessing above basic to intermediate competency. Specifically for information literacy most students demonstrated an above intermediate competency where for communication the majority was only above basic. Safety and problem solving had very similar profiles where again most students felt that they had only basic competency in these areas. Content creation was the area where the students felt least competent with 20 participants $(30 \%$ of the respondents) indicating an above intermediate competency. It is interesting to note that $23(40 \%)$ individuals were rated at both extremes with $4(7 \%)$ declaring no skills and $19(33 \%)$ declaring high levels of skills in some of the competency areas. It is also noteworthy that a significant number of 14 (25\%) students declared a high level of competency in the information digital literacy area.

When students were asked how they find out about new digital technologies, they reported that they discover new technologies primarily from friends and family $(38 \%)$, traditional media $(23 \%)$, online digital sources $(23 \%)$, library services $(9 \%)$, as part of their course at university (24\%), as part of their CPD at work (1\%) and professional or other specialist network $(1 \%)$.

Participants were also asked to identify their preferences of using select technologies by reporting on 
their use in their private, academic and work lives. Figure 5 - Technology Use below shows the utilisation of different types of technologies for formal/informal learning, research, work and in their personal life. Laptop computers are the predominant technology used in formal learning (52), with desktop computers (39), tablets (37) and smartphones (27) also commonly used. For informal learning laptop computers (51) are the most prevalent technology, followed by smart phones (46), tablets (41) and desktop computers (33) which are also being used extensively. Laptops are the most utilised devices in research (55) while tablet devices (42) and smartphones (40) are also regularly used. In the workplace laptops (38) are used by the majority of the respondents, desktops (29) by more than half of the participants, whereas smartphones (28) and tablets (24) are used by a minority. In their private lives, students seem to use a larger variety of technologies where smart phones (54), laptops (54), tablets (48) and digital photographic cameras (45) are used by the majority or the respondents. It is also interesting to note that some students have never used netbook computers (38), e-readers (33), mobile telephones without internet access (23), digital videocameras (20), mp3 players (15), digital cameras (8), mobile telephones with internet access (7), desktop computers (7), and tablet devices (5).

\subsection{The Sustainable Electronic Assessment (SEA) Project Qualitative Results}

Twelve weeks after the nursing students were given the tablets and completed the questionnaire they were asked to consider their digital literacy learning and development cycle and critically self-reflect and document their experiences of using mobile tablet digital-technologies. The top three areas emerging from the analysis of their tablet technology use in their private, academic and work lives can be seen in Table 3 - Diary Analysis Top Three Categories below.

\section{Table 3 - Diary Analysis Top Three Categories}

\begin{tabular}{|cccccc|}
\hline Private & & Academic & \multicolumn{3}{c|}{ Work } \\
\hline Communication & 11 & Experience & 12 & Experience & 10 \\
Usability & 11 & Usage & 11 & Communication & 8 \\
Experience & 9 & Information & 8 & Organisation & 8 \\
\hline
\end{tabular}

Initial analysis of the reflective diaries showed that in their private lives students are concerned with communication (11), usability (11), and experience (9). Social networking and communicating with friends and family when travelling or being on the move was one of the most appreciated affordances of technology. Students also use mobile digital technologies to access systems for carrying out everyday activities including communication and interacting with the university. They expect a seamless experience when accessing systems from their smart phones or tablets and expect to be supported when things do not work properly.

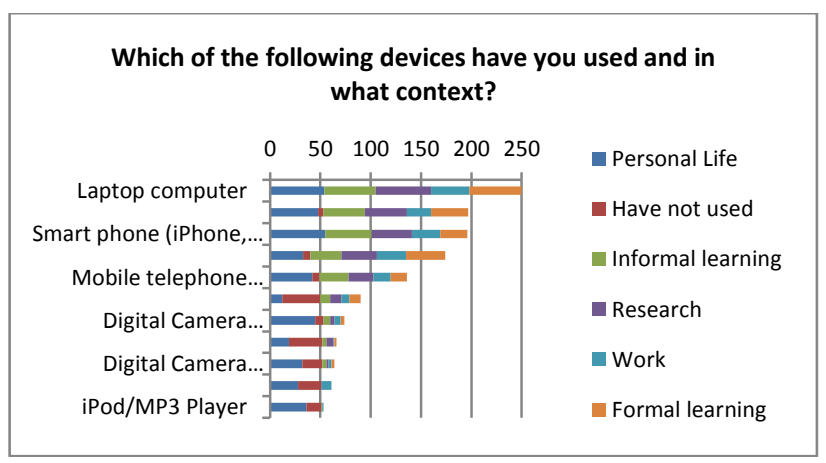

Figure 5 - Technology Use

In academic life they were concerned with experience (12), usage (11) and information (8). Most participants admitted that technology engagement for higher education study is a necessity and that they generally felt comfortable in using more than one type of technology. Tablet and smart phone use was widespread, and although some individuals admitted they were lacking the necessary skills for making effective use, they were willing to acquire the missing competences and skills. The main usage-patterns included the use of subject-specific applications to acquire knowledge, revising the PowerPoint handouts from the VLE, using single sign-on to access the university IT infrastructure, using tablet applications for note taking, accessing university information and timetabling, and e-submission of the assessment nurse competencies. From an information perspective mobile technologies are used for exam revisions, online information retrieval including books, journals and websites that enable them in their studies. Eight students emphasised the value of using tablet devices within lectures to broaden their understanding, check facts and definitions or review and further research difficult concepts.

In work life experience (10), communication (8), and organisation (8) are the top three categories of concern. There is consensus that mobile technologies are becoming increasingly pervasive in all aspects of everyday life including work and usage in the workplace. Participants generally felt comfortable with using the tablet devices for work and they drew examples on how these tablets were successfully used for data entry in workplace settings such as restaurants. The participants also reported that similar applications of technology could potentially change their work attitudes. From a communication perspective they generally found it useful to have access to technology when in clinical placements as they often needed to access information and/or communicate with the university and their tutors. Examples of organisational 
implications of technology-use in the workplace include the use of mobile devices with a variety of applications, such as using the calendar functions, reminders, or taking notes. One participant reflected, '... for patients for their doctors' visits, and their families' visits'; while another reported the use of social media as tools for publishing and managing rotas.

\subsection{The Classroom Technology-enhanced Learning Design Student Experience}

The student experience questionnaire included five questions aiming to evaluate the student experience of undertaking technology-enhanced, activity-based learning by using tablet devices in the classroom. Participants indicated their agreement or disagreement on a four-step scale that ranged from strongly agree, agree, disagree and strongly disagree with a discreet option of not answering. In order to better visualise the participants' perceptions the four distinct steps have been merged into two according to agreement or disagreement, with the not answering option reported separately. The results can be seen in Figure $6-$ The Student Experience of Technology-enhanced Learning Designs below.

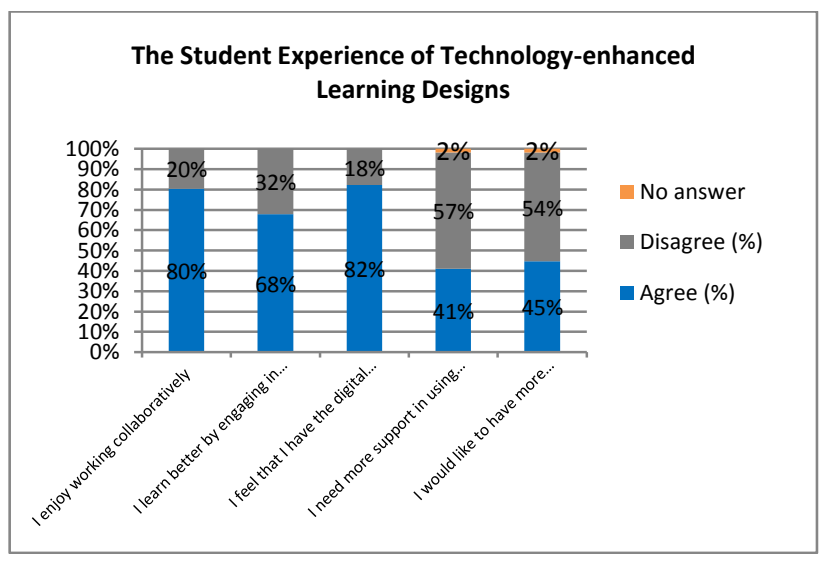

\section{Figure 6 - The Student Experience of Technology- enhanced Learning Designs}

The vast majority of students $(80 \%)$ stated that they enjoy working collaboratively with a considerable majority $(68 \%)$ reporting that they learn better when engaging in activity-based learning. Most students $(82 \%)$ felt confident that they possessed the digital skills required to complete the activities given. More than half of the participants $(57 \%)$ said that they do not require any additional support in using technologies, but a significant minority (41\%) stated that they would benefit from additional support. Finally, on the question of whether they would like more opportunities to learn collaboratively and participate in technology-enhanced, activity-based learning, more than half (54\%) said that they would not, but a sizeable minority $(45 \%)$ said that they would.

\section{Discussion}

Students across both case-study groups seemed to be reasonably comfortable in using technologies to communicate, learn, research and generally engage with them in a number of ways. As individuals on average they showed a command of above-basic digital competences located at the borderline of intermediate. At the same time the group seemed less comfortable in the areas of content creation, safety and problem solving, and more competent in information management and communication. Although at the individual level there is significant variance of digital capability, it must be stressed that the purpose of this research was the consideration of group dynamics as a broad-brush approach for the optimisation of teaching. This type of analysis is of interest for the optimisation of teaching when considering the development of digital skills and competences in learning and teaching. Interestingly, the frequency distribution of the participants' digital competences could reveal clusters (or sub-groups) of students with similar digital-capability potentials. The individual 'outliers' could be clustered into sub-groups of students who lacked digital skills and others who had expert profiles. This method of analysis offers possibilities for early identification of students with advanced, or indeed, lacking in, essential digital skills. This offers potential in the classroom for early intervention in the latter case; and further development and utilisation of those with existing advanced skills. For teaching, it may be possible to construct more balanced groups, and thus scaffold informal learning of digital skills by considering Vygotskyian [9] ideas of 'the more capable peer'.

From a technology-use perspective, students selfreported significant use of technologies in their private, academic and work lives. Specifically, the technologies were used in their personal lives for informal learning, research, work and formal learning; these were (in descending order from the most utilised to the least): a) laptop computers, tablet devices, smart phones, desktop computers, mobile phones with internet access, netbook computers, digital-photography cameras, e-readers, digital-videography cameras, mobile phones without internet access and mp3/ipod players. It is interesting to note that the three most used devices for formal learning were laptops, desktops and tablets where for informal learning these were laptops, smart phones and tablets. When conducting research the three most used devices by students were laptops, tablets and smart phones. This indicates a trend for increased use of mobile devices and in particular for the use of tablets and smartphones for learning. It is also interesting to note that desktop computers are only prevalent in the formal learning scenario, something that is likely to be happening because they are institutionally provided in the formal academic 
environments. The top three devices used in the private lives of the students were smart phones, laptops and tablets. The data suggests that the types of technologies used by students are changing and moving away from the traditional desktop computer towards mobile technologies, such as smart phones, laptops and tablet devices. For this reason, academic institutions should modernise their digital-device provision to include and support mobile devices. More importantly institutions should be prepared to utilise mobile technologies for learning and teaching, provide appropriate learning material for the use of these devices and acknowledge that these technologies are increasingly used by the students.

In the first case-study (SEA pilot project) reflective diaries where students self-reflected on their digitalliteracy affordances were collected. The research diaries that were collected for documenting the intricate details of the individual competences, skills and attitudes allowed for the appreciation of the main areas of focus of each student. It seems that students face academic life as a part of their 'everyday' life, and practice placements as their 'workplace'. What matters to them is the way they individually use technology to achieve their own aims in their own private, academic and work lives; this offers insights for the academics seeking to support their teaching. However, these distinctions were arbitrary as most students reflected from their individual circumstances and experiences. The analysis of the reflective diaries clearly demonstrated that students do not readily experiment, differentiate or select technologies according to the requirements of the task, but they usually revert to a small range of technologies they are already familiar with and they have used in the past. This is true even if other more appropriate technologies have been made available to them. A possible explanation is that learning how to use new technologies is still acting as a barrier and students will only attempt to use unfamiliar technologies if they are explicitly required to do so.

In the second case-study (The Technology-enhanced Learning Design Project) the student experience of utilising tablet devices to perform learning tasks in the classroom and beyond was evaluated. This project piloted a process for embedding digital literacies into curriculumdelivery by utilising activity-based, technology-enhanced learning designs. The majority of students enjoyed working collaboratively and recognised that they learn better when engaging in activity-based learning. Most of them felt comfortable because their level of digital skills was sufficient to complete the activities. Over half of the students thought that they did not require any further support in using digital technologies, but a significant minority thought otherwise. Finally, on the question whether they would like to have more opportunities to learn collaboratively and participate in activity-based, technology-enhanced learning just over the half replied negatively, with a sizeable minority replying positively. Overall, students seemed to enjoy learning together, found the activities beneficial for their learning and were confident about their digital skills. The group seemed to be split on whether they would need more technology support and whether they would appreciate to be given more opportunities for this type of technology-enhanced learning. A possible explanation for this contradicting behaviour could be the fact that some of the participants feel intimidated and uncomfortable when using new, unfamiliar technologies for the first time.

\section{Conclusions}

The case studies documented in this paper explored the utilisation of tablet devices in the assessment of studentnurse clinical competences in practice (The Sustainable Electronic Assessment Project (SEA)) and, when they were used for activity-based learning in the classroom, for utilisation of technology-enhanced learning designs (The Classroom Technology-enhanced Learning Design Project). In both case studies a snap-shot of the digitalcapability potential of the participants was established by issuing a self-assessment questionnaire. The questionnaire toolkit was based on the EU DIGCOMP framework and produced quantitative, metric data corresponding to the digital competences, skills and attitudes of the participants. Following a multi-method approach additional qualitative data was gathered and the student experience was documented.

The metrics offer robust descriptors of digital competence and, when combined with an analysis of technology-use, student experience and diary analysis suggest types of technologies preferred for private, workplace and academic contexts of learning. These tools and framework enable academics and related professionals to assess the digital literacy potential of groups and individuals. For example the self-assessment tools could be used to establish a baseline of the digital capability of staff and students.

A pragmatist view of developing the digital capabilities of students and staff dictates the establishment of digital practices in education in a way that is conducive to learning, but also realistic in what is expected in the discipline. From a curriculum enhancement perspective the generic nature of the framework allows for the customisation of the examples to express digital literacy in terms that are relevant and familiar to the discipline. Through utilisation of a design approach academics can tailor their teaching and create learning experiences that deliver digital competence in an area of interest within the context of the academic curriculum. The thresholds and examples could be customised to meet the needs of the discipline and thus, to ensure that the curriculum intrinsically develops in digital literacy together with the capabilities of students that are expected within the field of study or profession. There are close links to the employability and skills development agenda developed in parallel across academia and in consultation with external stakeholders of a discipline or profession.

Further work will include the modelling of a curriculum-development process for embedding digital 
literacies into curriculum delivery through the utilisation of technology-enhanced, activity-based learning designs; the findings so far present potential for reconceptualisation of the curricula for the forthcoming intake of nursing students.

\section{Acknowledgements.}

We would like to thank all of our participants for committing their time to participate in our research project and for their insightful contributions.

\section{References}

[1] Evangelinos, G., AND Holley, D. (2015) A Qualitative Exploration of the DIGCOMP Digital Competence Framework: Attitudes of students, academics and administrative staff in the health faculty of a UK HEI. EAI Endorsed Transactions on e-Learning, 2(6): p.e1. DOI: 10.4108/el.2.6.e1.

[2] Evangelinos, G. AND Holley, D. (2014a) A Qualitative Exploration of the EU Digital Competence (DIGCOMP) Framework: A Case Study Within Healthcare Education. In:, E-Learning, E-Education, and Online-Training (ELEOT) First International Conference, Lecture Notes of the Institute for Computer Sciences, Social Informatics and Telecommunications Engineering, Washington DC, USA, 18-20 September 2014, G. Vincenti, A. Bucciero and C. Vaz de Carvalho, eds., Cham: Springer International Publishing, pp.85-92 DOI: 10.1007/978-3-319-132938_11.

[3] Evangelinos, G. AND Holley, D. (2014) Developing a Digital Competence Self-assessment Toolkit for Nursing Students. In: From Education to Employment and Meaningful Work with ICT E-learning at Work and the Workplace. Electronic Proceedings of the EDEN 2014 Annual Conference, European Distance and E-learning Network, Zagreb, Croatia, 10-13 June 2014, pp. 206-212. WWW: http://hdl.handle.net/10540/333373.

[4] Dalziel, J., Conole, G., Wills, S., Walker, S., Bennett, S., Dobozy, E., CAmeron, L., Badilescu-Buga, E. And Bower, M. (2013) The Larnaca Declaration on Learning Design - 2013. [online] Larnaca, Cyprus, Available at: $<$ http://www.larnacadeclaration.org $>$ [Accessed 30 Nov. 2015].

[5] FERRARI, A. (2013) DIGCOMP: A Framework for Developing and Understanding Digital Competence in Europe. [online] Luxembourg: Publications Office of the European Union Available at: $\langle$ http://ftp.jrc.es/EURdoc/JRC83167.pdf> [Accessed 03 Apr. 2015].

[6] Glaser, B.G., And Strauss, A.L. (2009) The Discovery of Grounded Theory: Strategies for Qualitative Research. New Brunswick, NJ, US: Transaction Publishers.

[7] Miles, M. And Huberman, M. (1994) Qualitative Data Analysis (2nd edition). Beverley Hills, CA: Sage.

[8] Guest, G., Namey, E. And Queen, E. (2012) Applied Thematic Analysis. Thousand Oaks, California: Sage Publications, Inc.

[9] Vygotsky, L.S. (1978) Mind in Society. The development of higher psychological processes (Cole, M., Ed.). Cambridge MA: Harvard University Press (Original work published 1930). 